\title{
Long non-coding RNA MIAT regulates ox-LDL-induced cell proliferation, migration and invasion by miR-641/STIM1 axis in human vascular smooth muscle cells
}

\author{
Gang $\mathrm{Ma}^{1}$, Shuting $\mathrm{Bi}^{1}$ and Pengfei Zhang ${ }^{2,3^{*}}$ (i)
}

\begin{abstract}
Background: Atherosclerosis (AS) is a primary cause of coronary heart and vascular diseases. Long non-coding RNAs (IncRNAs) are indicated to regulate AS progression. This study aimed to reveal the biological roles of IncRNA myocardial infarction associated transcript (MIAT) in oxidized low-density lipoprotein (ox-LDL)-induced human vascular smooth muscle cells (VSMCs).
\end{abstract}

Methods: The RNA levels of MIAT, microRNA-641 (miR-641) and stromal interaction molecule 1 (STIM1) were detected by quantitative real-time polymerase chain reaction (qRT-PCR). The protein levels were determined by western blot analysis. Cell proliferation was assessed by cell colony formation and DNA content quantitation assays. Cell migration and invasion were demonstrated by wound-healing and transwell assays. The putative binding relationships between miR-641 and MIAT or STIM1 were predicted by starbase online database, and identified by dual-luciferase reporter and RNA immunoprecipitation assays.

Results: MIAT and STIM1 expression were substantially upregulated, whereas miR-641 expression was downregulated in ox-LDL-induced VSMCs compared with control groups. Functionally, MIAT silencing attenuated oxLDL-induced cell proliferation, migration and invasion in VSMCs; however, these effects were impaired by miR-641 inhibitor. STIM1 overexpression also restrained miR-641-mediated impacts on cell proliferation and metastasis under ox-LDL. Mechanistically, MIAT acted as a sponge for miR-641, and miR-641 was associated with STIM1.

Conclusions: MIAT silencing hindered ox-LDL-induced cell proliferation, migration and invasion by downregulating STIM1 expression through binding to miR-641 in VSMCs. The mechanism provided us with a new target for AS therapy.

Keywords: AS, MIAT, miR-641, STIM1

*Correspondence: feilantianshi@163.com

2 Department of Cardiac Surgery, Central Hospital Affiliated to Shandong First Medical University, No.105, Jiefang Road, Jinan 250013, Shandong, People's Republic of China

Full list of author information is available at the end of the article

\section{Background}

Atherosclerosis (AS), an inflammatory disease, is a major cause of vascular diseases, including myocardial infarction, ischemic stroke and cardiovascular disease [1]. AS is featured by lipid deposition and contains fat-rich macrophages $(\mathrm{m} \varnothing)$ [2]. Multiple types of cells were revealed to take part in the pathogenesis of AS, such as vascular smooth muscle cells (VSMCs), endothelial cells (ECs) and 
mø [3]. Recent review revealed that SMCs regulated the production of many matrix proteins and promoted the development of the inflammatory response to lipid, suggesting the importance of SMCs in AS progression [4]. Besides, previous research explained that oxidized lowdensity lipoprotein (ox-LDL) was a vital risk factor for AS [5]. Thus, profoundly revealing the mechanism underlying ox-LDL-induced abnormal transformation of VSMCs can provide us with reliable targets for AS therapy.

Long noncoding RNA (lncRNA) is a non-protein-coding RNA with at least 200 base pairs in size, featured by high conservatism [6]. LncRNA can transfer messages and guide molecule to ribonucleoprotein complexes, thereby playing important roles in biological processes [7]. An increasing number of researches revealed that lncRNAs were involved in the progression of various diseases, including AS [8-10]. LncRNA myocardial infarction associated transcript (MIAT) was also revealed to mediate AS process. As reported, MIAT could inhibit efferocytosis via upregulating clusters of differentiation 47 (CD47) through binding to microRNA-149-5p (miR149-5p) [11]. MIAT also had ability to modulate diabetes mellitus-caused microvascular dysfunction [12]. However, there were few studies on the development of AS mediated by MIAT $[13,14]$.

MiRNAs are a category of noncoding RNAs with about 20 nucleotides in size, and regulate gene expression via targeting their noncoding sequences [15]. At present, miRNAs have been unveiled to play crucial parts in modulating cardiovascular cell functions, such as protecting against cardiac dysfunction after cerebral ischemia-reperfusion, inhibiting autophagy, and regulating cardiomyocyte growth [16-18]. Another miRNA, miR-641, only was reported to participate in the regulation of cancer progression. For example, Kong et al. indicated that miR-641 was under-expressed in lung cancer cells, and repressed cell proliferation [19]. In the study of Li and his colleagues, we found the cancer-promoting role of miR641 in pancreatic cancer by associating with LINC01963 [20]. Stromal interaction molecule 1 (STIM1), an important factor in regulating calcium channels, can dramatically affect intracellular $\mathrm{Ca}^{2+}[21]$. Researches unveiled that STIM1 was widely expressed in nonexcitable cells, including VSMCs [22]. He et al. showed that STIM1 silencing repressed cell apoptosis and reduced intracellular $\mathrm{Ca}^{2+}$ accumulation in cardiomyocytes [23]. $\mathrm{Xu}$ and his colleagues reported that resveratrol improved cardiac functional recovery via repressing STIM1-mediated regulation of intracellular $\mathrm{Ca}^{2+}$ [24]. Therefore, STIM1 was important for function of multiple cell types. Based on the above data, miR-641/STIM1 were hypothesized to participate in the regulatory mechanism of MIAT in AS, and whether miR-641/STIM1 pathway was responsible for the molecular mechanism by which MIAT regulated AS development needed to be explored.

The study aimed to investigate the therapeutic target for AS and reveal the role of MIAT in ox-LDL-induced VSMCs and the inner molecular mechanism.

\section{Methods \\ Cell culture and storage}

Human aorta vascular smooth muscle cells (VSMCs) were purchased from Procell (Wuhan, China) and grown in F12K medium (Procell, Wuhan, China) with $10 \%$ fetal bovine serum (FBS; Procell, Wuhan, China) and $1 \%$ penicillin/streptomycin (Procell, Wuhan, China) at $37^{\circ} \mathrm{C}$ in an incubator with $5 \% \mathrm{CO}_{2}$.

\section{Plasmid construction, oligonucleotide synthesis and cell transfection}

The small interfering RNA against MIAT (si-MIAT), the mimic of miR-641 (miR-641 mimic), the inhibitor of miR641 (miR-641 inhibitor) and controls (si-NC, miR-NC mimic and miR-NC inhibitor) were provided by GenePharma (Shanghai, China). The overexpression plasmids of MIAT (oe-MIAT) and STIM1 (pcDNA-STIM1) as well as their controls (Vector and pcDNA-NC) were built by Geneseed (Guangzhou, China). Lipofectamine 2000 (Thermo Fisher, Waltham, MA, USA) was employed for cell transfection based on the instructions of manufacturer. The sequences of oligonucleotides were si-MIAT 5'-GCATTTGGTTTCAGTTCTT-3', miR-641 mimic 5'-AAAGACAUAGGAUAGAGUCACCUC-3', miR-641 inhibitor 5'-GAGGUGACUCUAUCCUAUGUCUUU -3', si-NC 5'-GCAGTTGTTACTGTTTCTT-3', miR-NC mimic 5'-UUUGUACUACACAAAAGUACUG-3' ${ }^{\prime}$ and miR-NC inhibitor 5'-CAGUACUUUUGUGUAGUA CAAA-3'.

\section{3-(4,5-Dimethylthazol-2-yl)-2,5-diphenyltetrazolium bromide (MTT) assay}

VSMCs were diluted in F12K medium (Procell, Wuhan, China) and grown in 96-well plates for $16 \mathrm{~h}$. Then, cells were incubated with various concentrations $(0,25,50$, 75 and $100 \mu \mathrm{g} / \mathrm{mL}$ ) of ox-LDL (Yeasen, Shanghai, China) for $24 \mathrm{~h}$ or $50 \mu \mathrm{g} / \mathrm{mL}$ ox-LDL for different time $(0,12,24$, 36 and $48 \mathrm{~h}$ ). After that, MTT reagent (Solarbio, Beijing, China) was incubated with cells for $4 \mathrm{~h}$. Cell supernatant was discarded and dimethyl sulfoxide (Sigma, St. Louis, MO, USA) was used to dissolve formazan. The cell viability was determined by assessing the output of wavelength at $490 \mathrm{~nm}$ with a Varioskan LUX Multimode microplate reader (Thermo Fisher, Waltham, MA, USA). 


\section{Quantitative real-time polymerase chain reaction (qRT-PCR)}

Cultured VSMCs were collected and lysed with TransZol (TransGen, Beijing, China). RNA was isolated using an RNAsimple kit (Tiangen, Beijing, China). Then, cDNA was synthesized with a FastKing RT Kit (Tiangen, Beijing, China) or MicroRNA Reverse Transcription Kit (Thermo Fisher, Waltham, MA, USA). For determining the expression levels of MIAT, miR-641 and STIM1, SuperReal PreMix Color (Tiangen, Beijing, China) was mixed with synthesized cDNA and primers, and added into a 96-well IQ5 thermocycler (Bio-Rad, Hercules, CA, USA). After that, Data were assessed by the $2^{-\Delta \Delta \mathrm{Ct}}$ method. Glyceraldehyde 3-phosphate dehydrogenase (GAPDH) and U6 acted as controls. The sequences of forward and reverse primers were MIAT $5^{\prime}$-GTGGCTCAGGAGTGCTTC-3' and $5^{\prime}$-ACTTGC CCAGGGTTGTAG-3'; miR-641 5'-ACACTCCAG CTGGGGAGGTGACTCTATCCTAT-3' and $5^{\prime}$-TGG TGTCGTGGAGTCG-3'; STIM1 5'-TTGGATTCT TCCCGTTCT- $3^{\prime}$ and $5^{\prime}$-CTGGGCTGGAGTCTGTTT $-3^{\prime}$; GAPDH 5'-GGTCACCAGGGCTGCTTT-3' and 5'-GGAAGATGGTGATGGGATT-3'; U6 5'-CTCGCT TCGGCAGCACA- $3^{\prime}$ and $5^{\prime}$-AACGCTTCACGAATT TGCGT-3'.

\section{Cell colony formation assay}

VSMCs were seeded in 6-well plates for $16 \mathrm{~h}$ and treated with $50 \mu \mathrm{g} / \mathrm{mL}$ ox-LDL (Yeasen, Shanghai, China). Twenty-four hours later, si-MIAT, miR-641 inhibitor, miR-641 mimic or pcDNA-STIM1 was transfected into the cells at $\sim 70 \%$ confluence with controls according to the defined purposes. Then, cells were cultured for 2 weeks. F12K medium (Procell, Wuhan, China) was renewed every 3 days during culture. The forming colonies were immobilized with paraformaldehyde (Sigma, St. Louis, MO, USA) and then dyed with crystal violet (Sigma, St. Louis, MO, USA). Cell colony-forming ability was determined by assessing the number of colonies. A colony was deemed when cell numbers over 50 .

\section{DNA content quantitation assay}

Cell cycle was detected by DNA content quantitation assay. In short, cultured VSMCs were collected and fixed with cold ethanol (Millipore, Bradford, MA, USA). Cells were precipitated by centrifugation at $300 \mathrm{~g}$ for $5 \mathrm{~min}$, and incubated with RNase A (Solarbio, Beijing, China) at $37{ }^{\circ} \mathrm{C}$ for $30 \mathrm{~min}$. After that, propidium iodide (PI; Solarbio, Beijing, China) was employed to stain cells at $4{ }^{\circ} \mathrm{C}$ for $30 \mathrm{~min}$. Samples were assessed by a flow cytometry (Thermo Fisher, Waltham, MA, USA).

\section{Wound-healing assay}

VSMCs were grown in 6-well plates and treated with different purposes. Cells were cultured until its confluence reached about $100 \%$. Cell wounds were created with $10-\mu \mathrm{L}$ pipette tips and cells were then cultivated in FBSfree F12K medium (Procell, Wuhan, China). At $24 \mathrm{~h}$ after culture, the width of the wounds was measured under an inverted microscope (Nikon, Tokyo, Japan) with a $40(\times)$ magnification, and cell migratory ability was determined by analyzing wound width.

\section{Western blot analysis}

Cells were harvested and lysed with RIPA buffer (Sigma, St. Louis, MO, USA) possessing proteinase K (Millipore, Bradford, MA, USA). Then, proteins were denaturalized at $95{ }^{\circ} \mathrm{C}$, and lysates were loaded onto $12 \%$ bis-trisacrylamide gel (Thermo Fisher, Waltham, MA, USA) to separate proteins. The protein bands were transferred onto polyvinylidene fluoride membranes (Millipore, Bradford, MA, USA), which were then immersed in 5\% non-fat milk (Solarbio, Beijing, China). Subsequently, the membranes were incubated with anti-proliferating cell nuclear antigen (anti-PCNA) (1:1500; Affinity, Nanjing, China), anti-nuclear proliferation marker (anti-Ki-67) (1:1500; Affinity, Nanjing, China), anti-phospho-focal adhesion kinase (anti-p-FAK) (1:1000; Affinity, Nanjing, China), anti-Ago2 (1:1000; Affinity, Nanjing, China), anti-IgG (1:2000; Abcam, Cambridge, UK), anti-STIM1 (1:1500; Affinity, Nanjing, China) and anti-GAPDH (1:15,000, Affinity, Nanjing, China). The membranes were incubated with horseradish peroxidase-marked secondary antibody (1:5000; Affinity, Nanjing, China). The protein bands were presented with RapidStep ECL Reagent (Millipore, Bradford, MA, USA), and protein expression was determined by Image J software $(\mathrm{NIH}$, Bethesda, MD, USA).

\section{Transwell migration and invasion assays}

The migrated and invaded cells were determined by transwell chambers without or with Matrigel (Corning, Madison, New York, USA). In brief, cells were seeded in the upper chambers containing FBS-free F12K medium (Procell, Wuhan, China) after treated with ox-LDL, siMIAT, si-NC, miR-641 inhibitor, miR-NC inhibitor, miR-641 mimic, miR-NC mimics, pcDNA-STIM1 or pcDNA-NC. In the lower chambers, F12K medium containing 15\% FBS (Procell, Wuhan, China) was added. Twenty-four hours later, cell supernatant was discarded, and cells were incubated with paraformaldehyde (Sigma, St. Louis, MO, USA) and crystal violet (Sigma, St. Louis, MO, USA), respectively. Results were determined via counting the number of cells in the lower chambers 
under a microscope (Nikon, Tokyo, Japan) at a $100(\times)$ magnification.

\section{Dual-luciferase reporter assay}

The binding sites between miR-641 and MIAT or STIM1 were firstly assessed through starbase online database (http://starbase.sysu.edu.cn/agoClipRNA.php? source= mRNA). And the wild-type (WT) and mutant (MUT) plasmids of MIAT and the 3'-untranslated region (3'UTR) of STIM1 were built by Geneseed Co., Ltd. (Guangzhou, China), and named as WT-MIAT, WT-STIM1 3'UTR, MUT-MIAT and MUT-STIM1 3'UTR. Constructed plasmids and synthesized oligonucleotides were transfected into the VSMCs at $\sim 70 \%$ confluence using Lipofectamine 2000 (Thermo Fisher, Waltham, MA, USA). Post-culture of $48 \mathrm{~h}$, the cells were collected and lysed using lysis buffer (Promega, Madison, WI, USA). Luciferase activities were detected with a dual luciferase reporter assay kit (Promega; Madison, WI, USA). Renilla luciferase activity served as a control.

\section{RNA immunoprecipitation (RIP) assay}

MiR-641 mimic was transfected into VSMCs with miR$\mathrm{NC}$ mimics as a control. Post-transfection of $48 \mathrm{~h}$, the cells were collected and lysed with RIP lysis buffer (Millipore, Bradford, MA, USA) containing protease inhibitor (Millipore, Bradford, MA, USA). After that, lysates were incubated with the magnetic beads bound with antiAgo2 (RIP-Ago2; Abcam, Cambridge, UK) or anti-IgG (RIP-IgG; Abcam, Cambridge, UK). Twenty-four hours later, the magnetic beads were washed, and MIAT and STIM1 expression were determined by qRT-PCR.

\section{Statistical analysis}

Data derived from three independent duplicate tests were assessed by SPSS 21.0 software (IBM, Somers, NY, USA). Results were expressed as means \pm standard deviations (SD). Significant differences were compared with two-tailed Student's $t$-tests between the two groups or one-way analysis of variance (ANOVA) with Tukey's test among three or more groups. Statistical significance was defined when $P$ value $<0.05$.

\section{Results}

\section{MIAT expression was upregulated in ox-LDL-induced} VSMCs

In order to determine the impact of ox-LDL treatment on MIAT expression, the reasonable induction dose and induction time of ox-LDL for VSMCs were firstly determined. Result presented ox-LDL exposure (50, 75 and $100 \mu \mathrm{g} / \mathrm{mL}$ ) promoted cell viability in a dosedependent manner (Fig. 1a). Additionally, data showed that $50 \mu \mathrm{g} / \mathrm{mL}$ ox-LDL also facilitated cell viability in a time-dependent manner with a minimum incubation time of $24 \mathrm{~h}$ (Fig. 1b). Based on these data, VSMCs were incubated with $50 \mu \mathrm{g} / \mathrm{mL}$ ox-LDL for $24 \mathrm{~h}$ in further oxLDL-related study. The impacts of various concentrations of ox-LDL $(0,25,50,75$ and $100 \mu \mathrm{g} / \mathrm{mL})$ on MIAT expression were continued to be explored. Our data displayed MIAT expression was substantially upregulated in VSMCs treated by ox-LDL (50, 75 and $100 \mu \mathrm{g} / \mathrm{mL}$ ) in a concentration-dependent manner (Fig. 1c). Meanwhile, results exhibited $50 \mu \mathrm{g} / \mathrm{mL}$ ox-LDL increased MIAT expression after culture for $24 \mathrm{~h}$ (Fig. 1d). These data demonstrated that MIAT might be involved in ox-LDLinduced VSMC cell disorder.

\section{MIAT silencing attenuated ox-LDL-induced cell proliferation, migration and invasion in VSMCs}

To further determine whether MIAT participated in the regulation of ox-LDL-induced cell proliferation, migration and invasion, si-MIAT was transfected into oxLDL-induced VSMCs with control groups. Results firstly showed that MIAT knockdown impaired the promoting impact of ox-LDL on MIAT expression (Fig. 2a). Cell colony formation assay showed that ox-LDL treatment enhanced the colony-forming ability of VSMCs, whereas this impact was restored after MIAT silencing (Fig. 2b). Meanwhile, data presented that ox-LDL exposure promoted cell arrest in S phase, while this impact was hindered after downregulation of MIAT (Fig. 2c). To further explain the effect of MIAT silencing on ox-LDL-triggered cell proliferation, we detected the protein expression of proliferation-related PCNA and Ki-67. As shown in Fig. 2d, ox-LDL treatment upregulated the protein expression of PCNA and Ki-67; however, MIAT knockdown attenuated these effects. The above data suggested that MIAT silencing restored ox-LDL-triggered cell proliferation. Subsequently, western blot analysis presented that the protein expression of metastasis-related p-FAK was increased after treatment of ox-LDL, while the effect was impaired after transfection of si-MIAT (Fig. 2e). Also, the enhanced migratory and invasive capacities of VSMCs by ox-LDL were reversed after MIAT absence (Fig. 2f-h). The results from Fig. 2e-h indicated that MIAT silencing restored ox-LDL-triggered cell migration and invasion. Taken together, the above evidences demonstrated MIAT knockdown assuaged ox-LDL-induced cell disorders.

\section{MiR-641 was directly associated with MIAT and STIM1 in VSMCs}

The study continued to explore the underlying mechanism of MIAT in regulating ox-LDL-mediated VSMC process. According to the results predicted by starbase online database, we found that MIAT contained the 

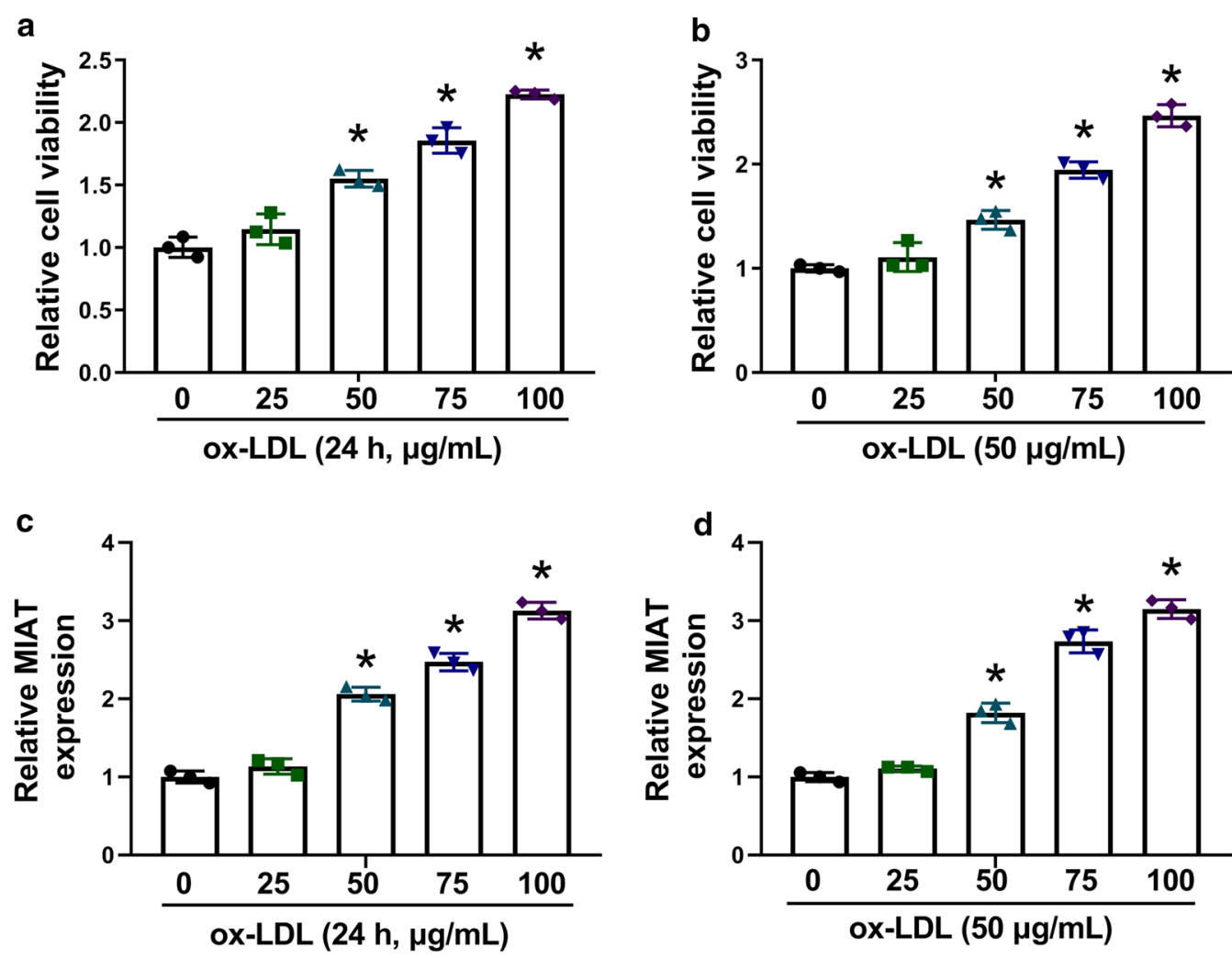

Fig. 1 MIAT expression was increased in ox-LDL-incubated VSMCs. a MTT assay was employed to demonstrate the impacts of various concentrations of ox-LDL $(0,25,50,75$ and $100 \mu \mathrm{g} / \mathrm{mL})$ on the viability of VSMCs at $24 \mathrm{~h}$ after incubation. $\mathbf{b}$ The effects of $50 \mu \mathrm{g} / \mathrm{mL}$ ox-LDL on the viability of VSMCs were demonstrated by MTT assay at various time points $(0,12,24,36$ and $48 \mathrm{~h})$ after incubation. c The impacts of various doses of ox-LDL $(0,25,50,75$ and $100 \mu \mathrm{g} / \mathrm{mL})$ on MIAT expression at $24 \mathrm{~h}$ after incubation were revealed by qRT-PCR. $\mathbf{d}$ The effects of $50 \mu \mathrm{g} / \mathrm{mL}$ ox-LDL on MIAT expression were determined by qRT-PCR at various time points $(0,12,24,36$ and $48 \mathrm{~h})$ after incubation. ${ }^{*} P<0.05$

binding sites of miR-641, which was further found to have complementary sites of STIM1 3'UTR (Fig. 3a, b). To further determine whether both MIAT and STIM1 were associated with miR-641, dual-luciferase reporter and RIP assays were employed. Before that, we detected the overexpression or knockdown efficiency of miR-641 mimic, miR-641 inhibitor, oe-MIAT and si-MIAT, and results were shown in Figure S1A-C. Then, as shown in Fig. $3 c, d$, the relative luciferase activity was significantly repressed in miR-641 mimic and WT-MIAT co-transfection group as well as in miR-641 mimic and WT-STIM1 3'UTR co-transfection group, whereas the relative luciferase activity had no obvious change in miR-641 mimic and MUT-MIAT co-transfection group or in miR-641 mimic and MUT-STIM1 3'UTR co-transfection group. Meanwhile, RIP assay presented both MIAT and STIM1 pulled down by RIP-Ago2 was significantly enriched in the VSMCs transfected with miR-641 mimic as compared with control groups (Fig. 3e). Additionally, it was found that miR-641 expression was significantly decreased after overexpression of MIAT, but upregulated by MIAT silencing (Fig. 3f). And qRT-PCR data showed that miR641 inhibitor substantially upregulated STIM1 protein expression, but miR-641 mimic downregulated STIM1 protein expression (Fig. 4g). The above results explained that miR-641 was directly associated with MIAT and STIM1 in VSMCs. Furthermore, data presented miR-641 expression was notably decreased and STIM1 protein expression was increased in ox-LDL-induced VSMCs (Fig. 3h, i), suggesting that miR-641 and STIM1 might participate in ox-LDL-induced VSMC disorders (Additional file 1).

MIAT silencing repressed ox-LDL-induced cell proliferation, migration and invasion by binding to miR-641 in VSMCs

Whether MIAT regulated ox-LDL-induced VSMC process by interacting with miR-641 was revealed in this part. Results initially exhibited MIAT silencing attenuated the repressive impact of ox-LDL exposure on miR-641 expression, whereas this impact was reversed after transfection of miR-641 inhibitor (Fig. 5a). Additionally, si-MIAT-mediated repressive impact on cell 


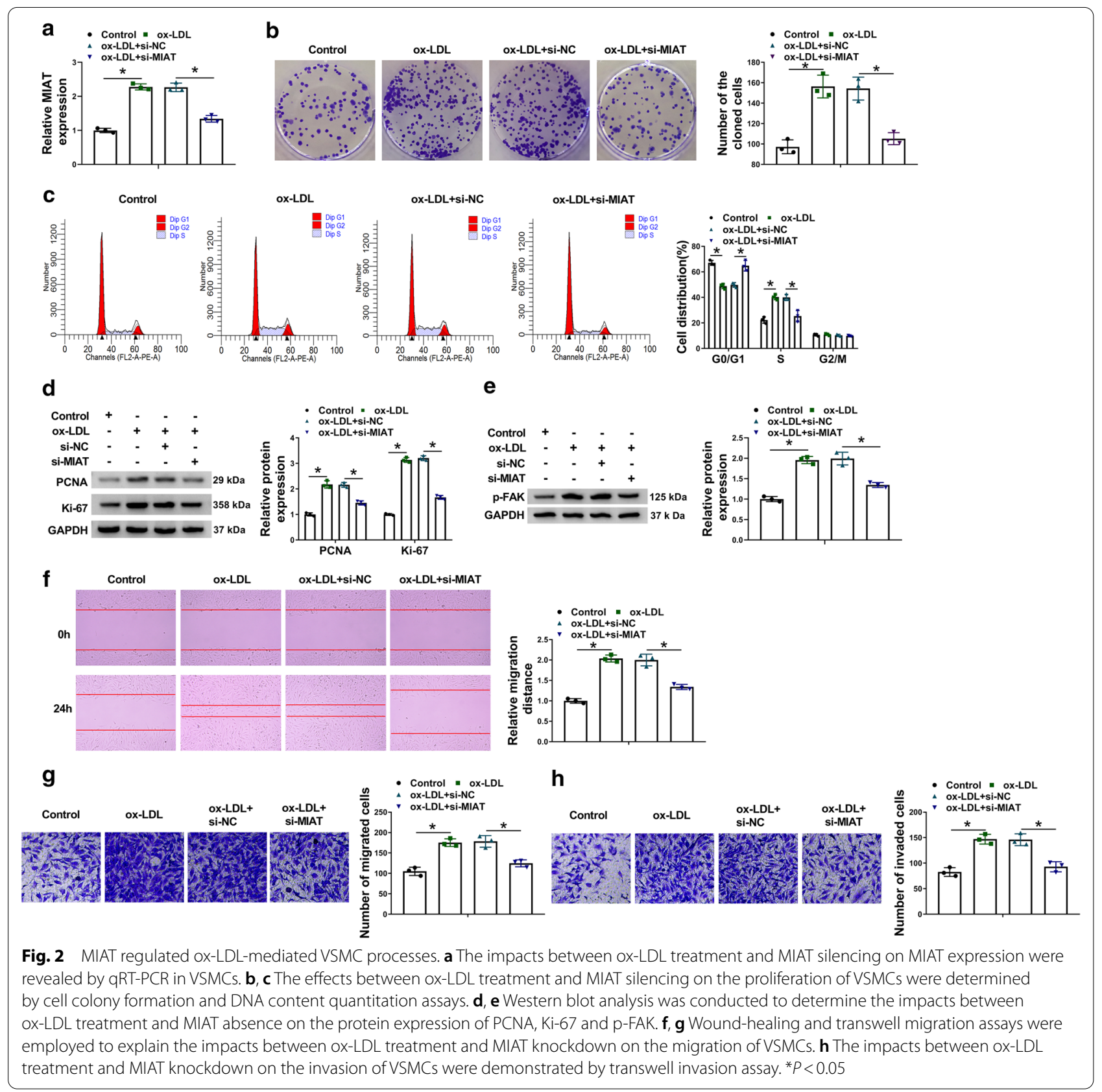

colony-forming ability was restored by miR-641 inhibitor in ox-LDL-induced VSMCs (Fig. 5b). DNA content quantitation assay also presented MIAT silencing promoted cell arrest in G0/G1 phase in ox-LDL-induced VSMCs, which was hindered after transfection of miR-641 inhibitor (Fig. 5c). Meanwhile, western blot analysis showed the protein expression of PCNA, Ki-67 and p-FAK was significantly downregulated after silencing of MIAT in ox-LDL-induced VSMCs, but these effects were hindered by miR-641 inhibitor (Fig. 5d-e). Furthermore, MIAT silencing repressed cell migration and invasion in oxLDL-induced VSMCs; however, these results were attenuated by miR-641 inhibitor (Fig. 5f-h). All in all, these data manifested that MIAT modulated ox-LDL-induced cell proliferation, migration and invasion by sponging miR-641 in VSMCs. 


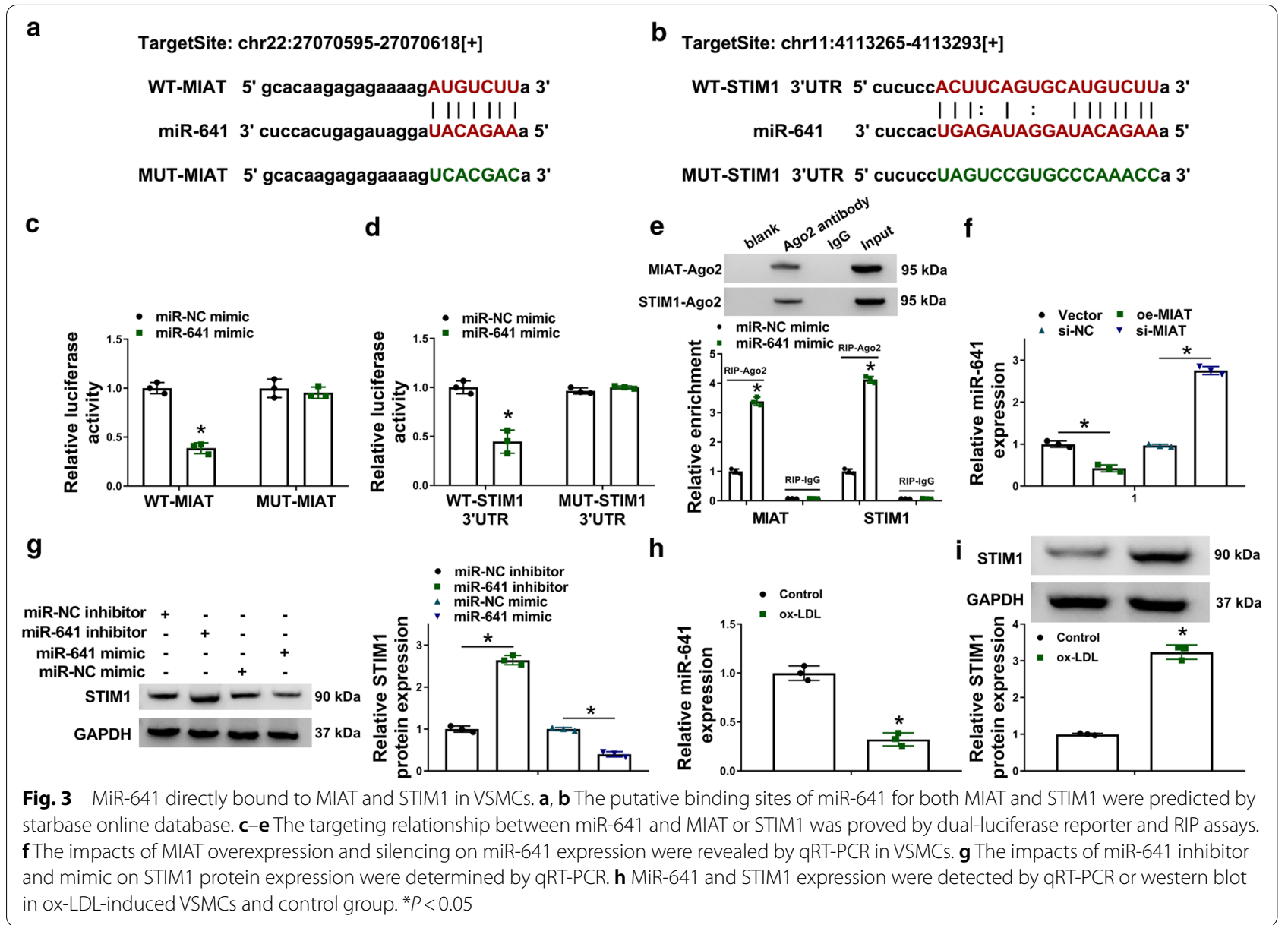

\section{MiR-641 repressed ox-LDL-induced cell proliferation,} migration and invasion by targeting STIM1 in VSMCs

Given the associated relationship between miR-641 and STIM1, whether miR-641 modulated ox-LDL-induced cell proliferation, migration and invasion by binding to STIM1 was further explored. Results firstly showed that miR-641 mimic attenuated the upregulating impact of ox-LDL treatment on STIM1 protein expression, whereas ectopic STIM1 expression hindered this impact (Fig. 4a). Additionally, the impacts of ox-LDL on cell colony-forming ability and $S$ phase cell arrest were impaired by miR-641 mimic, which was reversed after transfection of pcDNA-STIM1 (Fig. 4b, c). As expected, miR-641 mimic restored ox-LDL-induced promotion on the PCNA and Ki-67 protein levels, but STIM1 overexpression hindered these influences (Fig. 4a). Subsequently, miR-641 mimic-mediated downregulation of p-FAK protein expression under ox-LDL treatment was attenuated by ectopic STIM1 expression (Fig. 4d). Also, we found the enhanced migratory and invasive capacities of VSMCs by ox-LDL were restrained after miR641 mimic transfection; however, enforced expression of STIM1 abolished these impacts (Fig. 4e-g). Collectively, these findings explained that miR-641 repressed ox-LDL-induced cell proliferation, migration and invasion via interacting with STIM1.

\section{MIAT regulated STIM1 expression by associating with miR-641}

Whether MIAT controlled STIM1 expression by associating with miR-641 was continued to be revealed. To this end, si-MIAT and miR-641 inhibitor were co-transfected into VSMCs with control groups. Result showed that MIAT silencing significantly downregulated STIM1 protein expression, whereas this effect was restored after transfection of miR-641 inhibitor (Fig. 6a). On the contrary, MIAT overexpression increased STIM1 protein expression, but this effect was restrained by miR-641 mimic (Fig. 6b). These results demonstrated that MIAT could modulate STIM1 expression by binding to miR641. Thus, we came a conclusion that ox-LDL treatment upregulated MIAT expression, which further sponged miR-641 to induce STIM1, thereby promoting cell proliferation, migration and invasion (Fig. 6c). 


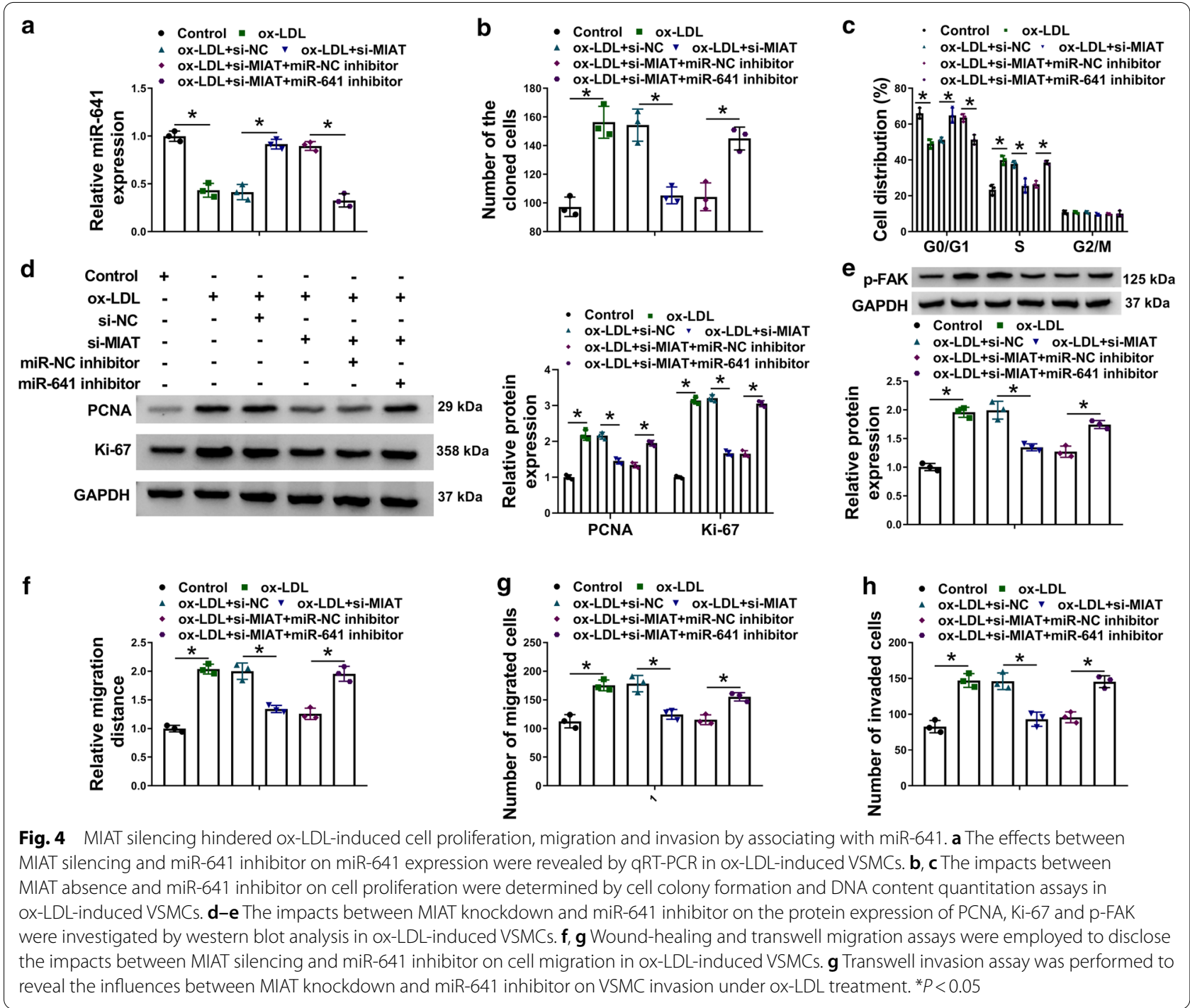

\section{Discussion}

Although many achievements have been created in revealing the pathogenesis of AS, AS still poses a heavy threat to human health [25]. Previous researches showed that lots of lncRNAs participated in regulating the process of cardiovascular diseases, including AS [26, 27]. As reported, lnc00113 could accelerate cell proliferation and migration via mediating phosphoinositide 3-kinase (PI3K)/protein kinase B (Akt)/mTOR pathway in AS [28]. Xia et al. indicated lncRNA gm 4418 accelerated cell apoptosis and deteriorated hypertensive cerebral arteriosclerosis [29]. In another example, retinal non-coding RNA3 (RNCR3) knockdown aggravated hypercholesterolemia and repressed the proliferative and migratory abilities of VSMCs [30]. Herein, we found MIAT silencing repressed cell proliferation, migration and invasion by regulating miR-641/STIM1 axis in ox-LDL-induced VSMCs.

Previous studies presented that MIAT participated in the regulation of AS progression. For example, MIAT promoted atherosclerotic plaques formation, angiogenesis and inflammation, and upregulated lipid content $[11,13]$. Zhong et al. explained MIAT accelerated cell proliferation, but restrained cell apoptosis via sponging miR-181b [31]. Additionally, it was found that MIAT was augmented and enhanced cell proliferative and metastatic capacities in ox-LDL-stimulated VSMCs [14, 32]. In this paper, we also found MIAT was elevated in oxLDL-induced VSMCs. MIAT absence restored ox-LDLinduced proliferation, migration and invasion in VSMCs. As reported, PCNA takes part in many aspects of DNA replication and is regarded as an important regulator of 

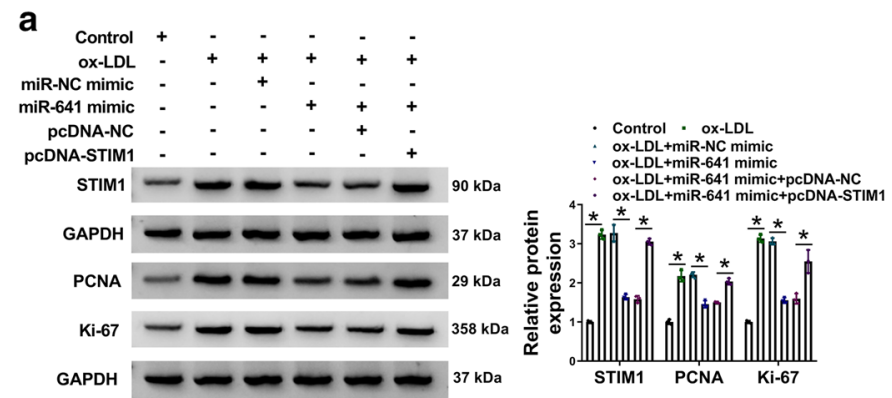

C

d

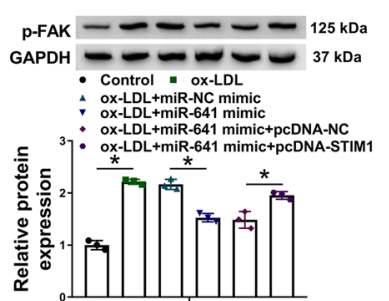

e

- Control = ox-LDL

4 ox-LDL+miR-NC mimic - ox-LDL+miR-641 mimictpcDNA-NC

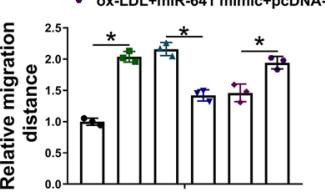

b

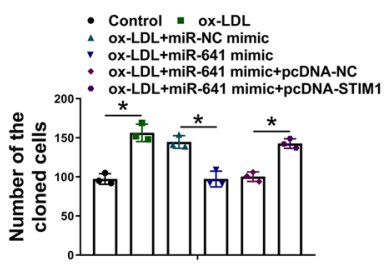

f

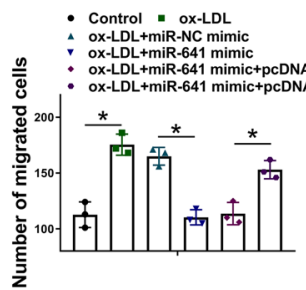

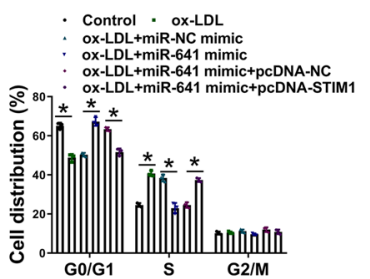

g

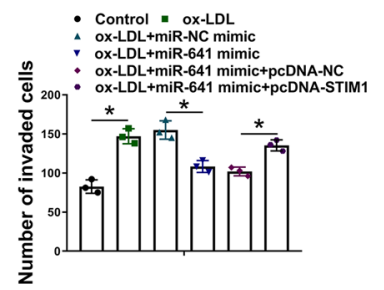

Fig. 5 MiR-641 hindered ox-LDL-induced cell proliferation, migration and invasion via interacting with STIM1. a, d The impacts between miR-641 and STIM1 overexpression on STIM1, PCNA, Ki-67 and p-FAK protein expression under ox-LDL treatment were detected by western blot analysis in VSMCs. b, c The impacts between miR-641 mimic and STIM1 overexpression on cell proliferation under ox-LDL treatment were revealed by cell colony formation and DNA content quantitation assays in VSMCs. e, f The impacts between miR-641 and STIM1 overexpression on cell migration under ox-LDL treatment were investigated by wound-healing and transwell migration assays in VSMCs. $\mathbf{g}$ The effects between miR-641 mimic and STIM1 overexpression on cell invasion under ox-LDL treatment were revealed by transwell invasion assay in VSMCs. ${ }^{*} P<0.05$

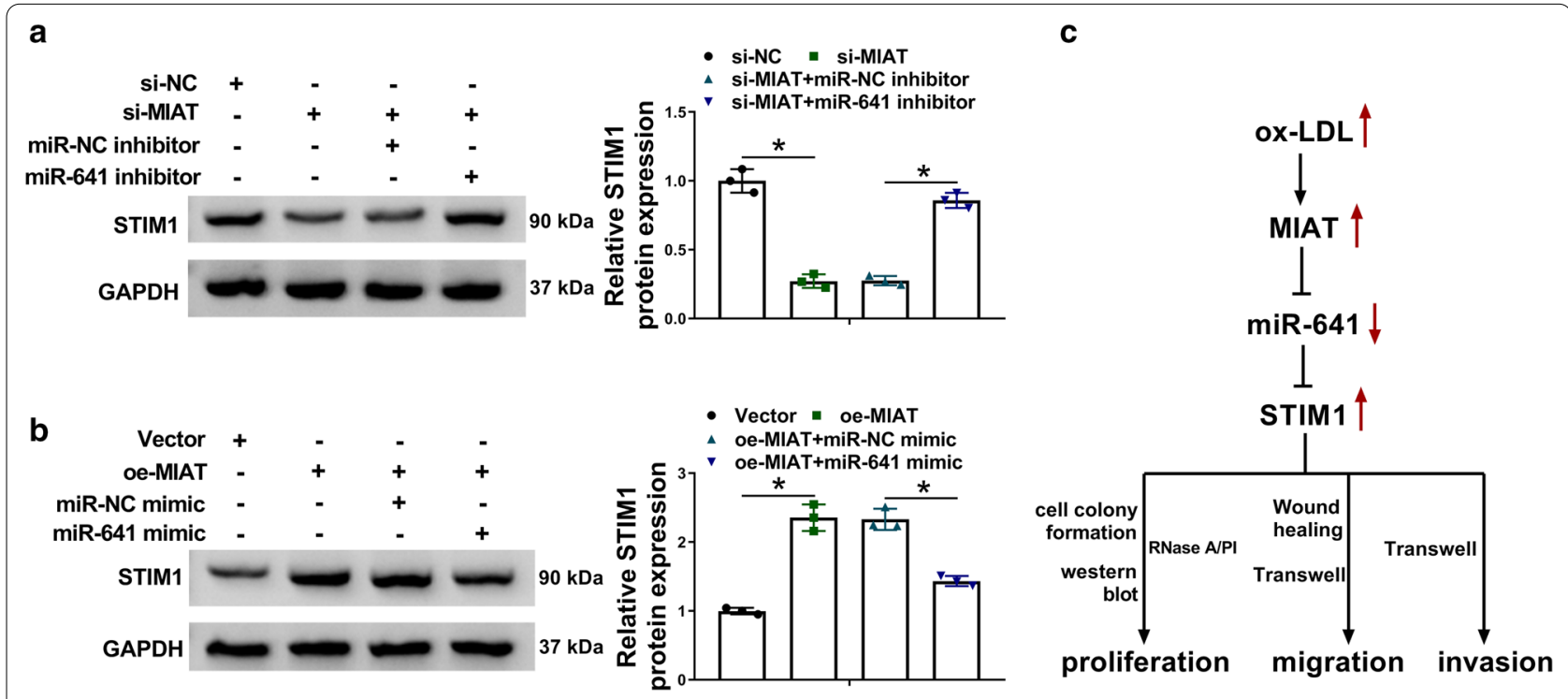

Fig. 6 MIAT modulated STIM1 expression by interacting with miR-641. a The impacts between MIAT silencing and miR-641 inhibitor on STIM1 protein expression were determined by western blot analysis in VSMCs. b The impacts between MIAT overexpression and miR-641 mimic on STIM1 protein expression were determined by western blot analysis in VSMCS. c The schematic diagram of the mechanism underlying MIAT regulating ox-LDL-induced VSMC disorders. ${ }^{*} P<0.05$ 
vital events at the replication fork [33]; Ki-67 has a conserved leucine/arginine rich $\mathrm{C}$-terminusand that can bind to DNA, thereby promoting chromatin compaction [34]; FAK is a tyrosine kinase and can be recruited to sites of integrin clustering or focal adhesions, and its phosphorylation status is revealed to be correlated with cell metastasis [35]. In the study, we found MIAT absence also attenuated the promoting effects of ox-LDL on the protein expression of PCNA, Ki-67 and p-FAK. All these evidences suggested the repressing role of MIAT in ox-LDL-induced VSMC disorders. Considering that lncRNA-miRNA-mRNA network played a vital part in unveiling the pathogenesis of cardiovascular diseases [36], the miRNA and mRNA associated with MIAT were sought. Results exhibited that MIAT interacted with miR-641, which was further revealed to target STIM1.

Current researches revealed that miR-641 was closely correlated with disease progression. Researches indicated that miR-641 repressed cell proliferation in lung cancer [37] and gastric cancer [38]. Zhang et al. explained that miR-641 participated in osteoarthritis process by regulating extracellular matrix metabolism and inflammation [39]. Additionally, miR-641 was reported to repress cisplatin resistance and erlotinib sensitivity in lung cancer $[40,41]$. Here, the paper was the first one to report the role of miR-641 in AS process. We found miR-641 expression was decreased in ox-LDL-treated VSMCs, and miR-641 inhibitor restrained MIAT knockdownmediated impacts, which suggested that miR-641 served as a suppressor in AS evolution. Meanwhile, the evidences from our study suggested that MIAT regulated ox-LDL-induced development of VSMCs via interacting with miR-641.

$\mathrm{Ca}^{2+}$ influx into cells is commonly mediated by capacitative $\mathrm{Ca}^{2+}$ entry pathway, which is reported to be modulated by STIM (STIM1 and STIM2) as well as Orai proteins [42]. This finding implied that STIM1 played a crucial part in biological behaviors of various cells. Coincidently, we found STIM1 was a target gene of miR-641. Herein, STIM1 was overexpressed in ox-LDL-treated VSMCs. And ectopic STIM1 expression impaired miR641-mdiated effects on cell proliferation and metastasis in ox-LDL-stimulated VSMCs. The evidences from our research implicated STIM1 served as a promoter in the progression of ox-LDL-stimulated VSMCs, which was proved by the existed evidences $[43,44]$. In the meantime, the above data implied that miR-641 repressed the progression of VSMCs stimulated by ox-LDL via binding to STIM1. Given the associated relationships between MIAT and miR-641 as well as between miR-641 and STIM1, whether MIAT modulated STIM1 by binding to miR-641 was continued to be illustrated. Rescue experiments showed MIAT silencing significantly decreased
STIM1 protein expression, whereas miR-641 inhibitor attenuated this impact. Additionally, miR-641 mimic also restored the upregulating impact of MIAT overexpression on STIM1 protein expression. These evidences suggested MIAT could control STIM1 expression through associating with miR-641.

However, there are some limitations that should be considered when interpreting our findings. First, the study only focuses on the roles of MIAT in regulating ox-LDL-induced cell injury in vitro, and the in vivo data are lacking in the present research, which is expected to be explored in future. To address the question, we would study the biological role of MIAT using Apoe-/ - mouse. Additionally, ox-LDL-induced VSMC injury might not only attribute to MIAT/miR-641/ STIM1 pathway, and there might were other singling pathways, which needed to be explored by more assays and bioinformatics methods.

\section{Conclusions}

Collectively, MIAT knockdown hindered cell proliferation, migration and invasion in ox-LDL-treated VSMCs. MiR-641 inhibitor reversed MIAT silencingmediated action under ox-LDL treatment. Additionally, MIAT was associated with miR-641, which was further proved to target STIM1. STIM1 overexpression also attenuated miR-641-mediated effects on the process of VSMCs stimulated by ox-LDL. All in all, MIAT modulated ox-LDL-induced cell proliferation, migration and invasion via miR-641/STIM1 pathway in VSMCs. Our finding not only provides a new mechanism for unveiling the pathogenesis of AS, but also lays a foundation for studying lncRNA-directed AS therapy.

\section{Abbreviations}

AS: Atherosclerosis; MIAT: myocardial infarction associated transcript; VSMCs: vascular smooth muscle cells; PCNA: proliferating cell nuclear antigen.

\section{Supplementary Information}

The online version contains supplementary material available at https://doi. org/10.1186/s12872-021-02048-9.

Additional file 1. Figure S1: The expression of miR-641 and MIAT was detected by qRT-PCR. a The expression of miR-641 was determined by qRT-PCR in the VSMCs transfected with miR-NC mimic or miR-641 mimic b QRT-PCR was employed to detect miR-641 expression in the VSMCs transfected with miR-NC inhibitor or miR-641 inhibitor. c The effects of oeMIAT and si-MIAT on MIAT expression were checked by qRT-PCR in VSMCs

\section{Acknowledgements}

None. 


\section{Authors' contributions}

GM designed the study, analyzed the data and wrote the manuscript. SB performed the experiments. PZ analyzed the data. All authors have read and approved the manuscript.

\section{Funding}

There is no funding resource.

\section{Availability of data and materials}

All data generated and/or analyzed during the current study are available from the corresponding author on reasonable request.

\section{Declarations}

\section{Ethics approval and consent to participate}

Not applicable.

\section{Consent for publication}

Not applicable.

\section{competing interests}

The authors declare that they have no competing interest.

\section{Author details}

'Deptment of Cardiac Surgury, Zibo Central Hospital, Zibo 255036, Shandong, People's Republic of China. ${ }^{2}$ Department of Cardiac Surgery, Central Hospital Affiliated to Shandong First Medical University, No.105, Jiefang Road, Jinan 250013, Shandong, People's Republic of China. ${ }^{3}$ Department of Cardiac Surgery, Jinan Central Hospital Affiliated to Shandong University, Jinan, Shandong, People's Republic of China.

Received: 17 December 2020 Accepted: 5 May 2021

Published online: 20 May 2021

\section{References}

1. Geovanini GR, Libby P. Atherosclerosis and inflammation: overview and updates. Clin Sci (Lond). 2018;132:1243-52.

2. Libby P. Coronary artery injury and the biology of atherosclerosis: inflammation, thrombosis, and stabilization. Am J Cardiol. 2000;86:3J-8J; discussion 8J-9J.

3. Lusis AJ. Atherosclerosis. Nature. 2000;407:233-41.

4. Doran AC, Meller N, McNamara CA. Role of smooth muscle cells in the initiation and early progression of atherosclerosis. Arterioscler Thromb Vasc Biol. 2008:28:812-9.

5. Pirillo A, Norata GD, Catapano AL. LOX-1, OxLDL, and atherosclerosis. Mediators Inflamm. 2013;2013:152786.

6. Ma H, Hao Y, Dong X, Gong Q, Chen J, Zhang J, et al. Molecular mechanisms and function prediction of long noncoding RNA. Sci World J. 2012;2012:541786.

7. Chen L, Yang W, Guo Y, Chen W, Zheng P, Zeng J, et al. Exosomal IncRNA GAS5 regulates the apoptosis of macrophages and vascular endothelial cells in atherosclerosis. PLOS ONE. 2017;12:e0185406-6.

8. Zhang YX, Yuan J, Gao ZM, Zhang ZG. LncRNA TUC338 promotes invasion of lung cancer by activating MAPK pathway. Eur Rev Med Pharmacol Sci. 2018:22:443-9.

9. Wang J, Su Z, Lu S, Fu W, Liu Z, Jiang X, et al. LncRNA HOXA-AS2 and its molecular mechanisms in human cancer. Clin Chim Acta. 2018;485:229-33.

10. Shen $\mathrm{Z}$, Li H. Long non-coding RNA GAS5 knockdown facilitates proliferation and impedes apoptosis by regulating miR-128-3p/FBLN2 axis in ox-LDL-induced THP-1 cells. Clin Hemorheol Microcirc. 2020;77(2):153-64.

11. Ye ZM, Yang S, Xia YP, Hu RT, Chen S, Li BW, et al. LncRNA MIAT sponges miR-149-5p to inhibit efferocytosis in advanced atherosclerosis through CD47 upregulation. Cell Death Dis. 2019;10:138.

12. Yan B, Yao J, Liu JY, Li XM, Wang XQ, Li YJ, et al. IncRNA-MIAT regulates microvascular dysfunction by functioning as a competing endogenous RNA. Circ Res. 2015;116:1143-56.
13. Sun G, Li Y, Ji Z. Up-regulation of MIAT aggravates the atherosclerotic damage in atherosclerosis mice through the activation of PI3K/Akt signaling pathway. Drug Deliv. 2019;26:641-9.

14. Zhou Y, Ma W, Bian H, Chen Y, Li T, Shang D, et al. Long non-coding RNA MIAT/miR-148b/PAPPA axis modifies cell proliferation and migration in ox-LDL-induced human aorta vascular smooth muscle cells. Life Sci. 2020;256:117852

15. Bartel DP. MicroRNAs: genomics, biogenesis, mechanism, and function. Cell. 2004;116:281-97.

16. Zhang C. MicroRNAs: role in cardiovascular biology and disease. Clin Sci (Lond). 2008;114:699-706.

17. Ding S, Huang H, Xu Y, Zhu H, Zhong C. MiR-222 in cardiovascular diseases: physiology and pathology. Biomed Res Int. 2017;2017:4962426.

18. Feng $M, X u D$, Wang L. miR-26a inhibits atherosclerosis progression by targeting TRPC3. Cell Biosci. 2018;8:4.

19. Kong Q, Shu N, Li J, Xu N. miR-641 functions as a tumor suppressor by targeting MDM2 in human lung cancer. Oncol Res. 2018;26:735-41.

20. Li K, Han H, Gu W, Cao C, Zheng P. Long non-coding RNA LINC01963 inhibits progression of pancreatic carcinoma by targeting miR-641/ TMEFF2. Biomed Pharmacother. 2020;129:110346.

21. Perni $S$, Dynes $J L$, Yeromin $A V$, Cahalan MD, Franzini-Armstrong $C$. Nanoscale patterning of STIM1 and Orai1 during store-operated Ca2 + entry. Proc Natl Acad Sci USA. 2015;112:E5533-42.

22. Shi J, Miralles F, Kinet J-P, Birnbaumer L, Large WA, Albert AP. Evidence that Orai1 does not contribute to store-operated TRPC1 channels in vascular smooth muscle cells. Channels. 2017;11:329-39.

23. He F, Wu Q, Xu B, Wang X, Wu J, Huang L, et al. Suppression of Stim 1 reduced intracellular calcium concentration and attenuated hypoxia/ reoxygenation induced apoptosis in H9C2 cells. Biosci Rep. 2017;37.

24. Xu H, Cheng J, Wang X, Liu H, Wang S, Wu J, et al. Resveratrol pretreatment alleviates myocardial ischemia/reperfusion injury by inhibiting STIM1-mediated intracellular calcium accumulation. J Physiol Biochem. 2019;75:607-18.

25. Kattoor AJ, Kanuri SH, Mehta JL. Role of Ox-LDL and LOX-1 in atherogenesis. Curr Med Chem. 2019;26:1693-700.

26. Zhao Z, Sun W, Guo Z, Zhang J, Yu H, Liu B. Mechanisms of IncRNA/microRNA interactions in angiogenesis. Life Sci. 2020;254:116900.

27. Kumar S, Williams D, Sur S, Wang JY, Jo H. Role of flow-sensitive microRNAs and long noncoding RNAs in vascular dysfunction and atherosclerosis. Vascul Pharmacol. 2019;114:76-92.

28. Yao X, Yan C, Zhang L, Li Y, Wan Q. LncRNA ENST00113 promotes proliferation, survival, and migration by activating PI3K/Akt/mTOR signaling pathway in atherosclerosis. Medicine. 2018;97:e0473.

29. Xia WQ, Niu GZ, Yin CG, Lu S, Bu XY. Effects of IncRNA gm4419 on rats with hypertensive cerebral atherosclerosis through NF-KB pathway. Eur Rev Med Pharmacol Sci. 2019:23:10976-81.

30. Shan K, Jiang Q, Wang XQ, Wang YN, Yang H, Yao MD, et al. Role of long non-coding RNA-RNCR3 in atherosclerosis-related vascular dysfunction. Cell Death Dis. 2016;7:e2248.

31. Zhong X, Ma X, Zhang L, Li Y, Li Y, He R. MIAT promotes proliferation and hinders apoptosis by modulating miR-181b/STAT3 axis in ox-LDL-induced atherosclerosis cell models. Biomed Pharmacother. 2018;97:1078-85.

32. Ma H, Zheng L, Qin H, Wang W. MIAT knockdown inhibits cell proliferation, migration and invasion via miR-490-3p/ICAM1 axis in oxLDL-induced vascular smooth muscle cells. J Cardiovasc Pharmacol. 2020;76(5):617-26.

33. Boehm EM, Gildenberg MS, Washington MT. The many roles of PCNA in eukaryotic DNA replication. Enzymes. 2016;39:231-54.

34. Sobecki M, Mrouj K, Camasses A, Parisis N, Nicolas E, Llères D, et al. The cell proliferation antigen Ki-67 organises heterochromatin. eLife. 2016;5:e13722-2

35. Zeng X-Q, Li N, Ma L-L, Tseng Y-J, Zhao N-Q, Chen S-Y. Prognostic value of Focal Adhesion Kinase (FAK) in human solid carcinomas: a meta-analysis. PLOS ONE. 2016;11:e0162666-6.

36. Huang $Y$. The novel regulatory role of IncRNA-miRNA-mRNA axis in cardiovascular diseases. J Cell Mol Med. 2018;22:5768-75.

37. Fan YF, Yu ZP, Cui XY. IncRNA Colorectal Neoplasia Differentially Expressed (CRNDE) promotes proliferation and inhibits apoptosis in non-small cell lung cancer cells by regulating the miR-641/CDK6 Axis. Med Sci Monit. 2019;25:2745-55. 
38. Wang LW, Li XB, Liu Z, Zhao LH, Wang Y, Yue L. Long non-coding RNA OIP5-AS1 promotes proliferation of gastric cancer cells by targeting miR641. Eur Rev Med Pharmacol Sci. 2019;23:10776-84.

39. Zhang W, Zhang C, Hu C, Luo C, Zhong B, Yu X. Circular RNA-CDR1as acts as the sponge of microRNA-641 to promote osteoarthritis progression. J Inflamm (Lond). 2020;17:8.

40. Chen J, Cui JD, Guo XT, Cao X, Li Q. Increased expression of miR-641 contributes to erlotinib resistance in non-small-cell lung cancer cells by targeting NF1. Cancer Med. 2018;7:1394-403.

41. Zhao Y, Zheng R, Chen J, Ning D. CircRNA CDR1as/miR-641/HOXA9 pathway regulated stemness contributes to cisplatin resistance in non-small cell lung cancer (NSCLC). Cancer Cell Int. 2020;20:289.

42. Tanwar J, Trebak M, Motiani RK. Cardiovascular and hemostatic disorders: role of STIM and Orai proteins in vascular disorders. Adv Exp Med Biol. 2017;993:425-52.
43. Huang Z, Li P, Wu L, Zhang D, Du B, Liang C, et al. Hsa_circ_0029589 knockdown inhibits the proliferation, migration and invasion of vascular smooth muscle cells via regulating miR-214-3p and STIM1. Life Sci. 2020;259:118251.

44. Fang M, Li Y, Wu Y, Ning Z, Wang X, Li X. miR-185 silencing promotes the progression of atherosclerosis via targeting stromal interaction molecule 1. Cell Cycle. 2019;18:682-95.

\section{Publisher's Note}

Springer Nature remains neutral with regard to jurisdictional claims in published maps and institutional affiliations.
Ready to submit your research? Choose BMC and benefit from:

- fast, convenient online submission

- thorough peer review by experienced researchers in your field

- rapid publication on acceptance

- support for research data, including large and complex data types

- gold Open Access which fosters wider collaboration and increased citations

- maximum visibility for your research: over 100M website views per year

At BMC, research is always in progress.

Learn more biomedcentral.com/submissions 